\title{
Morphology and conductivity studies of a new solid polymer electrolyte: $(\mathrm{PEG})_{x} \mathrm{LiClO}_{4}$
}

\author{
TH JOYKUMAR SINGH and S V BHAT* \\ Department of Physics, Indian Institute of Science, Bangalore 560 012, India
}

MS received 14 August 2003; revised 1 October 2003

\begin{abstract}
A new solid polymer electrolyte, (PEG) $\mathrm{LiClO}_{4}$, consisting of poly(ethylene)glycol of molecular weight 2000 and $\mathrm{LiClO}_{4}$ was prepared and characterized using XRD, IR, SEM, DSC, NMR and impedance spectroscopy techniques. XRD and IR results show the formation of the polymer-salt complex. The samples with higher salt concentration are softer, less opaque and less smooth compared to the low salt concentration samples. DSC studies show an increase in the glass transition temperature and a decrease in the degree of crystallinity with increase in the salt concentration. Melting temperature of SPEs is lower than the pure PEG 2000. Room temperature ${ }^{1} \mathrm{H}$ and ${ }^{7} \mathrm{Li}$ NMR studies were also carried out for the $(\mathrm{PEG})_{x} \mathrm{LiClO}_{4} \mathrm{system}$. The ${ }^{1} \mathrm{H}$ linewidth decreases as salt concentration increases in a similar way to the decrease in the crystalline fraction and reaches a minimum at around $x=46$ and then increases. ${ }^{7} \mathrm{Li}$ linewidth was found to decrease first and then to slightly increase after reaching a minimum at $x=46$ signifying the highest mobility of Li ions for this composition. Room temperature conductivity first increases with salt concentration and reaches a maximum value $\left(\sigma=7.3 \times 10^{-7} \mathrm{~S} / \mathrm{cm}\right)$ at $x=46$ and subsequently decreases. The temperature dependence of the conductivity can be fitted to the Arrhenius and the VTF equations in different temperature ranges. The ionic conductivity reaches a high value of $\sim 10^{-4} \mathrm{~S} / \mathrm{cm}$ close to the melting temperature.
\end{abstract}

Keywords. Solid polymer electrolyte; $(\mathrm{PEG})_{x} \mathrm{LiClO}_{4}$; glass transition temperature; ${ }^{1} \mathrm{H}$ and ${ }^{7} \mathrm{Li}$ NMR spectra; ionic conductivity.

\section{Introduction}

The pioneering work in the field of solid polymer electrolytes (SPEs) was carried out by Wright and co-workers (Fenton et al 1973; Wright 1975), who reported ionic conductivity of the order of $10^{-5} \mathrm{~S} / \mathrm{cm}$ at $330 \mathrm{~K}$ in highly crystalline PEO-NaSCN complexes. Since then considerable progress has been made in this field (Whittingham et al 1988). In the past few decades, SPEs have gained considerable attention due to their interesting and wide application potentials in high energy density batteries, sensors and fuel cells (Armand et al 1978; Gray 1991). The morphological changes and the conductivity optimization with respect to the salt concentration are the main interest of the current research in SPEs. The study of variation of ionic conductivity with salt fraction is an interesting but formidable problem for both experimental and theoretical research. Most of these studies have been on high molecular weight [e.g. poly(ethylene) oxide, PEO, $\left(\sim 10^{6}\right)$ ] polymers complexed with alkali metal salts (lithium salts being the most studied (Ratner and Shriver 1988; Bruce and Vincent 1993)) and not much attention has been paid to the somewhat low molecular weight polymers ( mol. wt. 2000). It is known (Shi and Vincent

\footnotetext{
*Author for correspondence
}

1993) that polymers below a critical molecular weight $(\sim 3200)$ have different viscosity and diffusion behaviours. Bearing these facts in mind we have prepared a new SPE based on poly(ethylene) glycol (PEG) of molecular weight 2000 , complexed with lithium perchlorate salt. $\mathrm{LiClO}_{4}$ is chosen as it fulfils the electrochemical stability criteria (Vincent 1987) and has low lattice energy $\left(723 \mathrm{~kJ} / \mathrm{mol}^{-1}\right)$, which is favourable for the polymer-salt complex formation. Generally it is accepted that polymer-salt complexes have three distinct regions, viz. crystalline polymer, crystalline polymer-salt complex and amorphous polymersalt complex (Kelly et al 1984). Many of the host polymers, viz. PEO and PEG, used for complexing with certain low lattice energy salts to form the SPEs are partially crystalline in nature. It has been shown that it is mainly the amorphous regions that contribute to the observed ionic conductivity (Berthier et al 1983).

In this paper we report detailed studies on the synthesis and characterization of a new SPE consisting of PEG 2000 and $\mathrm{LiClO}_{4}$ using XRD, IR, SEM, DSC, NMR and ionic conductivity techniques.

\section{Experimental}

\subsection{Sample preparation}

The chemicals, poly(ethylene) glycol (PEG) of mol. wt. 2000 (Fluka), and $\mathrm{LiClO}_{4}$ (Fluka) were used without further 
purification. $\mathrm{LiClO}_{4}$ was dried in an oven overnight at $100-110^{\circ} \mathrm{C}$ to remove the moisture prior to the use, as it is hygroscopic in nature. The $(\mathrm{PEG})_{x} \mathrm{LiClO}_{4}$ samples $(x=15$, ..., 500, being the ratio of ether oxygens to $\mathrm{Li}^{+}$) were prepared by standard solution cast method using methanol (AR grade) as the common solvent. The complex solution was magnetically stirred for $7-8 \mathrm{~h}$ at room temperature and followed by another hour at $\sim 50^{\circ} \mathrm{C}$ in nitrogen atmosphere for the evaporation of the remaining solvent. The viscous solution was then poured into Teflon rings (diameter $\sim 7 \mathrm{~mm}$, thickness $\sim 1-2 \mathrm{~mm}$ ) and kept in a glove box under nitrogen atmosphere overnight and the solvent was allowed to evaporate slowly. The solidified sample was transferred to a vacuum desiccator for vacuum drying under continuous pumping. This was performed at room temperature, at a vacuum of $10^{-2}$ Torr for $4-5 \mathrm{~h}$.

\section{$2.2 X$-ray diffraction and infrared characterization}

The X-ray diffraction (XRD) patterns were recorded using a Scintag XDS 2000 diffractometer at a scan rate of $10 \%$ min. Samples were prepared on glass slides to make their insertion into the XRD machine easier. The area and the thickness of the films on the glass slides were kept the same for all the compositions so that comparison of intensities would be possible.

For the infrared (IR) recording small amounts of samples were pelletized (diameter $\sim 13 \mathrm{~mm}$, thickness $\sim 0.5 \mathrm{~mm}$ ) with $\mathrm{KBr}$ (AR grade) using a hydraulic press pelletizer (Graseby Specac Ltd., UK) at 5-7 tons. IR spectra were taken using the Bruker IFS-55 FTIR spectrometer in the range $500-4000 \mathrm{~cm}^{-1}$.

\subsection{SEM imaging}

The SEM (JEOL JSM-5600LV) pictures were taken to ascertain the surface morphology of the samples for various salt concentrations. The samples were gold coated using the Sputter Coater (Polaron SC 7610, UK) at $10 \mathrm{~mA}$ current and $10^{-2}$ Torr vacuum for $6 \mathrm{~min}$ prior to imaging.

\subsection{Thermal studies}

DSC measurements were carried out on a MDSC 2920 (TA Instruments, USA) in standard mode with nitrogen gas purging at the rate of $25 \mathrm{ml} / \mathrm{min} .10-12 \mathrm{mg}$ of the sample was used for DSC recording. Samples were heated to $100^{\circ} \mathrm{C}$ at the rate of $10^{\circ} \mathrm{C} / \mathrm{min}$ followed by cooling to $-100^{\circ} \mathrm{C}$. The recordings were done in heating as well as in cooling cycles.

\subsection{NMR experiment}

${ }^{1} \mathrm{H}$ and ${ }^{7} \mathrm{Li}$ NMR measurements were carried out on a Bruker DSX-300 spectrometer at room temperature. A single $90^{\circ}$ pulse of $4 \mu$ s was used with $0.5 \mathrm{~s}$ recycle delay. For the NMR experiments the films were cut into narrow strips and filled into $5 \mathrm{~mm}$ glass tubes in nitrogen atmosphere. The open end of the tube was closed using Teflon tape. The samples in the tubes were kept in a glove box till the time of the experiment.

\subsection{Conductivity measurement}

For the conductivity measurement the sample prepared in the Teflon ring was loaded to a specially designed spring press fit SS sample cell that exerts a small pressure on the sample to ensure good contact. Preparing the sample in a Teflon ring ensures the intactness of the sample during conductivity measurement. The conductivity experiments were carried out under continuous dry nitrogen flow to prevent the absorption of moisture as moisture affects the ionic conductivity drastically (Tanzella et al 1981; Weston and Steele 1982). The impedance measurements were carried out with a $500 \mathrm{mV}$ a.c. signal using a PAR 5210 vector lock-in amplifier in the frequency range $4 \mathrm{~Hz}-120 \mathrm{kHz}$. The measurements were made at every $5 \mathrm{~K}$ during heating using a Bruker VT-1000 temperature controller. After setting the desired temperature, the cell was kept at that temperature for 20-25 min to stabilize at the set value of temperature. The experiments were carried out in the temperature range $260-340 \mathrm{~K}$. All the experimental spectra were analysed using Boukamp's ‘equivalent software package (Boukamp 1989).

\section{Results and discussion}

\section{$3.1 \quad X R D$ and $I R$}

Figure 1 shows the XRD patterns for various salt compositions of the polymer-salt complexes, as well as that for pure PEG and pure $\mathrm{LiClO}_{4}$ salt. The two prominent peaks of PEG (at $2 \theta=19.2$ and $23.4^{\circ}$ ) are present in all the patterns indicating the presence of pure polymer in all. The $\mathrm{LiClO}_{4}$ peaks are seen in none of the polymer-salt complexes and this signifies that the $\mathrm{LiClO}_{4}$ solvates very well in PEG 2000 matrix. The intensity of the PEG peaks decreases at high salt concentrations. The diffraction peaks of the pure salt are absent in the polymer salt complexes indicating the absence of pure salt phase in these complexes.

Figure 2 shows the IR spectra for pure and polymersalt complex for $x=46$ salt composition. The change in IR spectra (disappearance of the peaks at 1968, 2166 and $2238 \mathrm{~cm}^{-1}$ ) confirms the formation of the polymer-salt complex.

\subsection{Sample morphology}

As a function of salt concentration the samples show interesting trends in other physical properties such as hygro- 
scopicity and mechanical strength as well. The high salt category with salt content higher than $2 \cdot 3$ mole $(x \leq 20)$ are brittle with rough surfaces and are very hygroscopic. The medium salt concentration category samples with salt content of $2 \cdot 3-0 \cdot 46$ mole $(20<x<100)$ are soft and moderately hygroscopic. The low salt concentration category samples with salt content below 0.46 mole $(x \geq 100)$ are harder than the films of high salt content and medium salt content categories but have smooth surfaces. These films are semi-transparent and not very hygroscopic unlike the high and medium salt concentration samples. The surface morphology of the high salt concentration system is rough compared with the low salt concentration samples as seen in the SEM images (see figure 3). The SEM of PEG shows the features of spherulites.

\subsection{Glass transition temperature and fractional crystallinity}

Figure 4 shows the DSC curves (endothermic) registered for pure PEG and (PEG) $)_{x} \mathrm{LiClO}_{4}(x=20,46$ and 200). The summary of the DSC results for the heating cycle (endothermic) is given in table 1. The signal to noise ratio of the signature of $T_{\mathrm{g}}$ in DSC is usually poor. Hence the $T_{\mathrm{g}}$ 's were determined by fitting the traces to appropriate function using the software available with the instrument. The fitting error is indicated in table 1 and is the uncertainty in the $T_{\mathrm{g}}$ values. An increase in the glass transition temperature, $T_{\mathrm{g}}$, with an increase in the salt concentration was observed and this signifies that the flexibility of the polymer chains decreased with an increase in the salt con- centration in the polymer system. This reduction in flexibility of chains is usually interpreted as being a result of the effects of an increase in intramolecular and intermolecular coordinations between coordinating sites on the same or different polymer chains caused by the ions acting as transient cross links (Le Nest et al 1988). $T_{\mathrm{g}}$ could not be found for lower salt concentration samples, as these samples are highly crystalline. Melting temperature, $T_{\mathrm{m}}$, of the system also decreases as the salt concentration increases.

The enthalpy of melting, $\Delta Q_{\mathrm{m}}$, is less for the SPEs in comparison with that of pure PEG 2000. The area under the curve for the melting endotherm is related to the crystallinity in the specimen. The ${ }^{1} \mathrm{H}$ NMR spectrum of the pure PEG used consists of a broad component and a narrow component as shown in figure 5. The two-component nature of ${ }^{1} \mathrm{H}$ NMR lineshape of PEG/PEO has been noted by many researchers (Hikichi and Furuichi 1965; Johansson et al 1992). It is generally accepted that the broad component is due to the crystalline regions and the narrow component corresponds to the amorphous regions. We have fitted a Gaussian curve to the broad signal and a Lorentzian to the narrow signal. Therefore, the ratio of the area of the broad component to the total area indicates the fraction of crystalline regions in the sample. The crystalline fraction of the pure PEG from the NMR result is $83 \%$. The heat of melting of PEG from the DSC was observed to be $168 \mathrm{~J} / \mathrm{g}$. The heat of melting of PEG was scaled up for $100 \%$ crystallinity and found to be $202.41 \mathrm{~J} / \mathrm{g}$. The degree of crystallinity, $X_{\mathrm{c}}$, of the $(\mathrm{PEG})_{x} \mathrm{LiClO}_{4}$ system is calculated from the ratio of the $\Delta Q_{\mathrm{m}}$ of these systems with the enthalpy of melting of $100 \%$ crystalline PEG

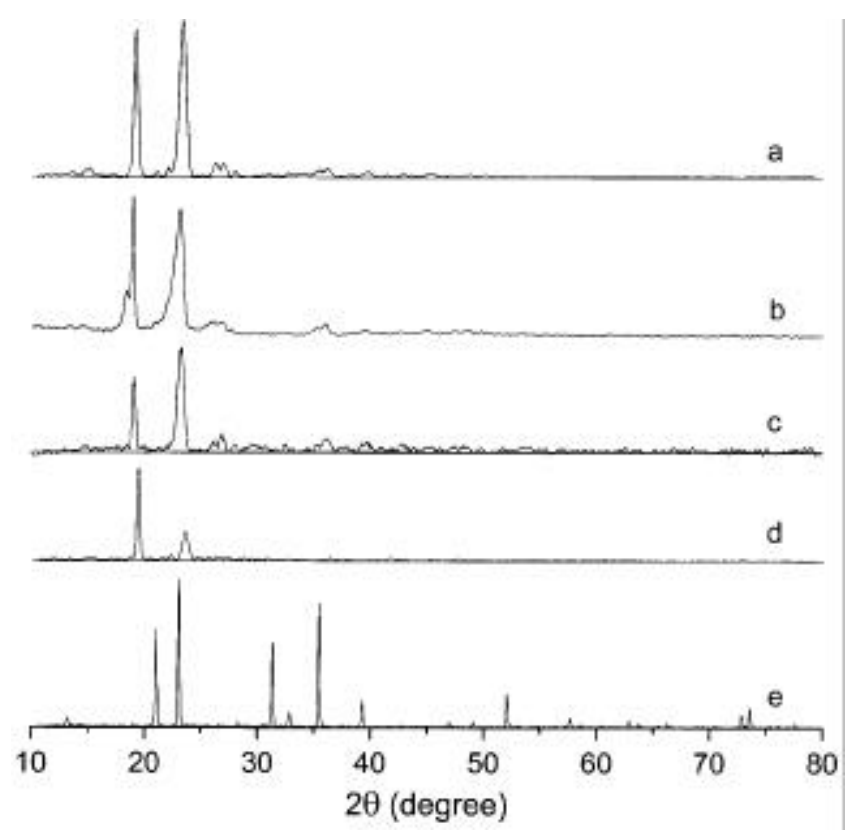

Figure 1. XRD pattern of a. PEG, b. $\mathrm{PEG}_{300} \mathrm{LiClO}_{4}$, c. $(\mathrm{PEG})_{46}$ $\mathrm{LiClO}_{4}$, d. $(\mathrm{PEG})_{20} \mathrm{LiClO}_{4}$ and e. $\mathrm{LiClO}_{4}$.

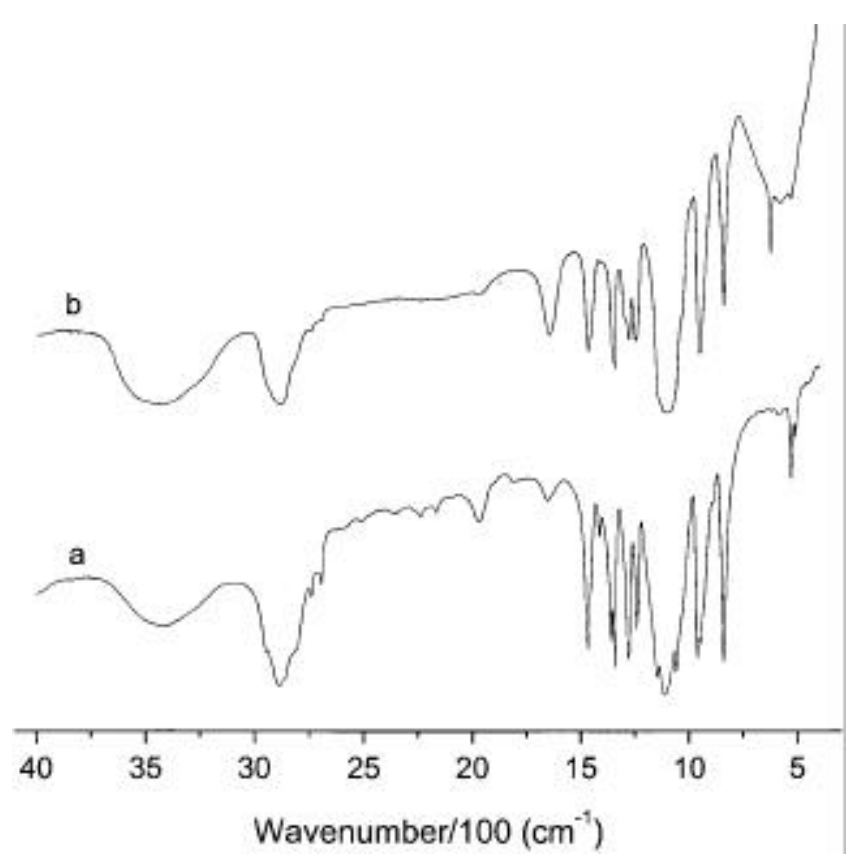

Figure 2. FT-IR plot for a. PEG and b. $(\mathrm{PEG})_{46} \mathrm{LiClO}_{4}$. 

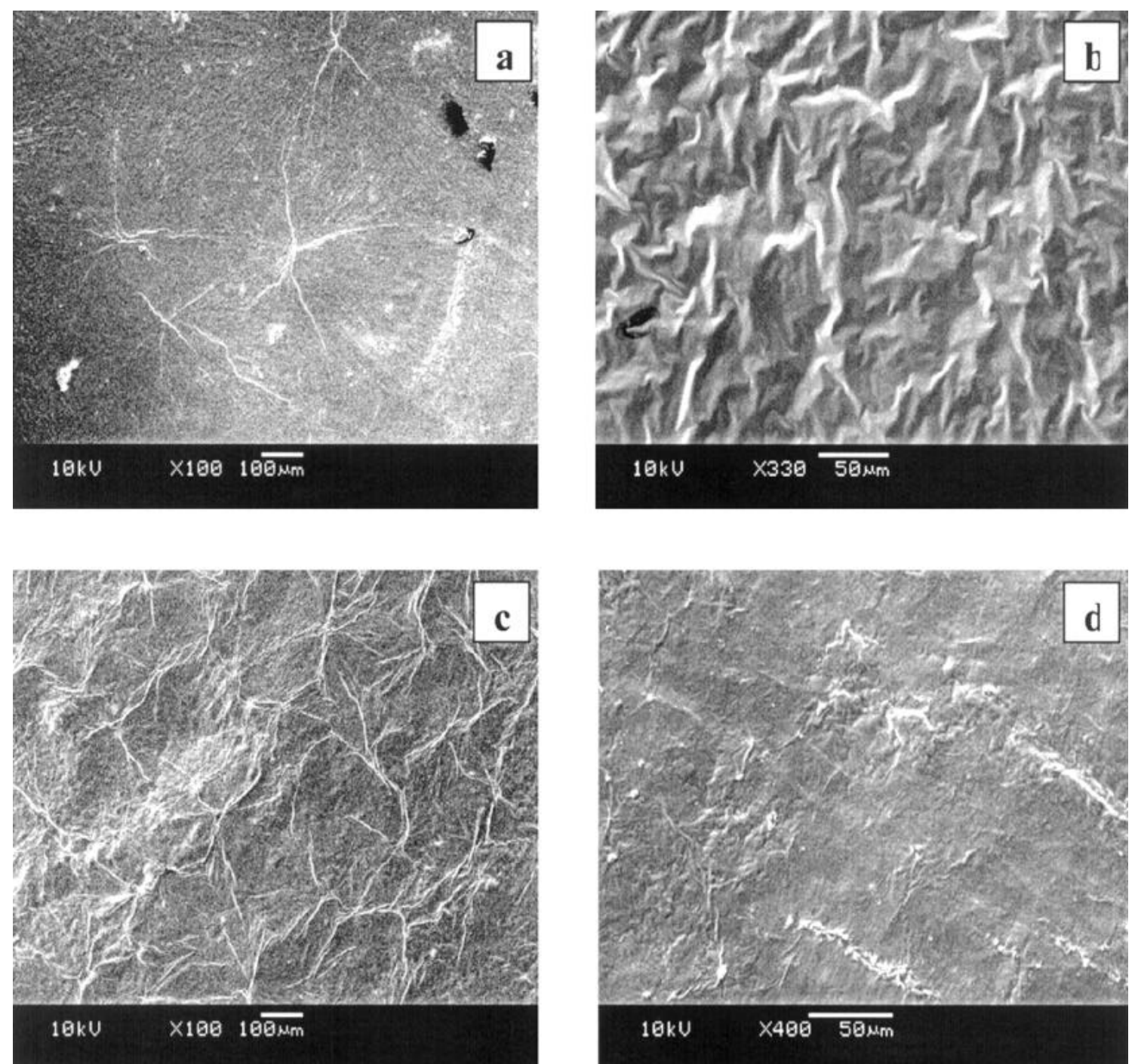

Figure 3. SEM images for a. PEG, b. $(\mathrm{PEG})_{20} \mathrm{LiClO}_{4}$, c. $(\mathrm{PEG})_{46} \mathrm{LiClO}_{4}$ and d. $(\mathrm{PEG})_{200} \mathrm{LiClO}_{4}$.

$\left(\Delta Q_{\mathrm{mPEG}}=202 \cdot 41 \mathrm{~J} / \mathrm{g}\right)$. This is the first ever report on using NMR and DSC techniques to calculate the $X_{c}$. Usually DSC is used to calculate the $X_{\mathrm{c}}$ by comparing the $\Delta Q_{\mathrm{m}}$ of the systems to that of the $100 \%$ pure and crystalline polymer like PEO (Li and Hsu 1984). For those polymers like PEG 2000, which are not $100 \%$ crystalline, this method is useful for calculating the $X_{\mathrm{c}} . X_{\mathrm{c}}$ decreases as the salt concentration increases (see figure 6). With an increase in $\mathrm{LiClO}_{4}$ salt concentration, $\Delta Q_{\mathrm{m}}$ decreased indicating a decrease in the $X_{\mathrm{c}}$ (Watanabe et al 1984). The reduction in crystalline fraction, $X_{\mathrm{c}}$, with increase in salt concentration can be attributed to the inhibition of crystallization by the salt.

\section{$3.4 \quad$ NMR results}

The ${ }^{1} \mathrm{H}$ NMR spectra of PEG 2000 consist of two overlapping signals. As mentioned earlier the broad component

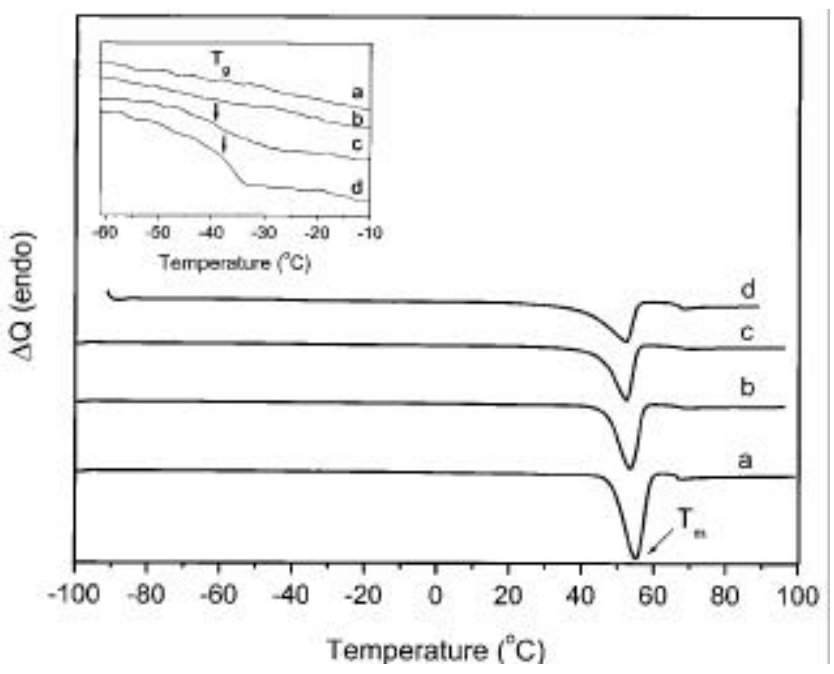

Figure 4. DSC traces (endothermic) obtained for a. pure PEG, b. $(\mathrm{PEG})_{200} \mathrm{LiClO}_{4}$, c. $(\mathrm{PEG})_{46} \mathrm{LiClO}_{4}$ and d. $(\mathrm{PEG})_{20} \mathrm{LiClO}_{4}$. 
Table 1. DSC results of $(\mathrm{PEG})_{x} \mathrm{LiClO}_{4}$.

\begin{tabular}{|c|c|c|c|c|}
\hline$x$ value & $\begin{array}{c}\text { Salt } \\
\text { content } \\
\text { (mole) }\end{array}$ & $\Delta H(\mathrm{~J} / \mathrm{g})$ & $T_{\mathrm{m}}\left({ }^{\circ} \mathrm{C}\right)$ & $T_{\mathrm{g}}\left({ }^{\circ} \mathrm{C}\right)$ \\
\hline 20 & $2 \cdot 300$ & $120 \cdot 3$ & $52 \cdot 3$ & $-36.4 \pm 1.0$ \\
\hline 30 & 1.533 & $135 \cdot 3$ & $53 \cdot 6$ & $-39.8 \pm 1.0$ \\
\hline 46 & $1 \cdot 000$ & $142 \cdot 4$ & $52 \cdot 3$ & $-38.8 \pm 1 \cdot 0$ \\
\hline 100 & $0 \cdot 460$ & $154 \cdot 7$ & $53 \cdot 8$ & - \\
\hline 200 & $0 \cdot 230$ & $155 \cdot 7$ & $53 \cdot 4$ & - \\
\hline 300 & $0 \cdot 153$ & $159 \cdot 8$ & $52 \cdot 8$ & - \\
\hline 500 & 0.092 & $161 \cdot 0$ & $52 \cdot 9$ & - \\
\hline PEG & 0 & $168 \cdot 0$ & $55 \cdot 2$ & - \\
\hline
\end{tabular}

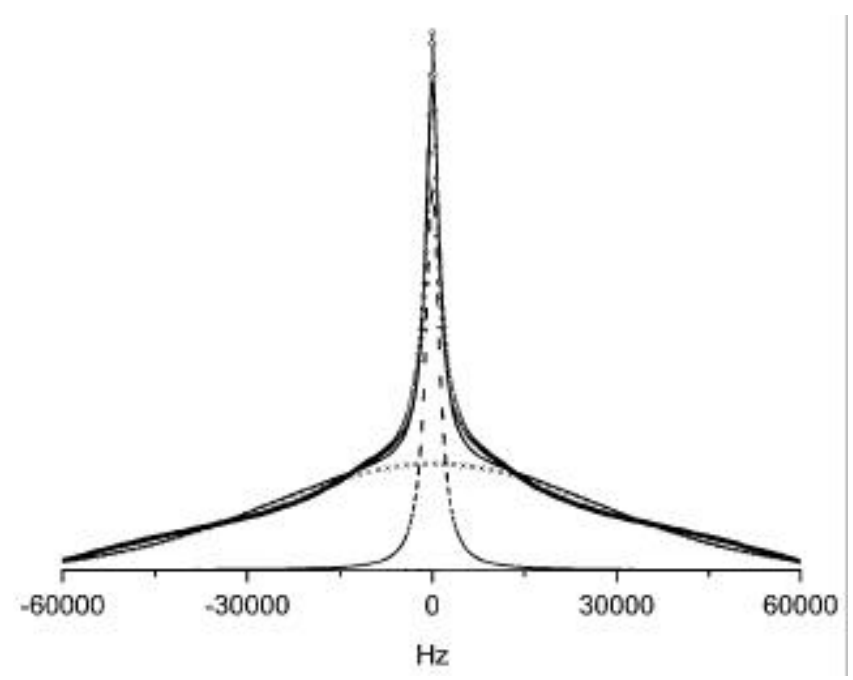

Figure 5. ${ }^{1} \mathrm{H}$ NMR spectrum of pure PEG at room temperature. The cross and dash lines are the fit to the Gaussian and the Lorentzian functions, respectively. The solid line is the resultant fit of both the functions and the circles are experimental points.

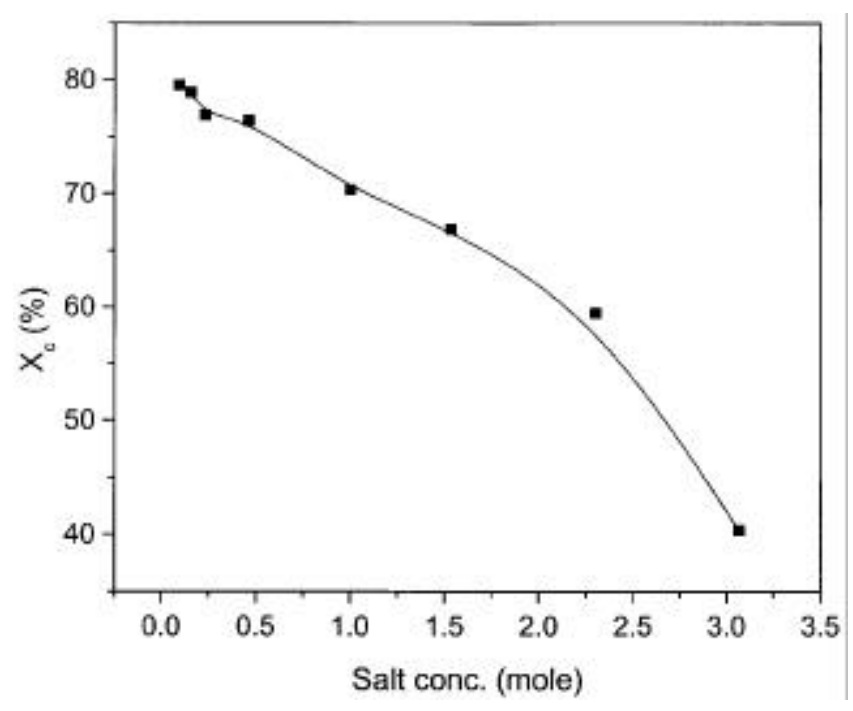

Figure 6. Crystallinity vs salt concentration for $(\mathrm{PEG})_{x} \mathrm{LiClO}_{4}$ system. The solid line is the guide to the eyes. is due to the crystalline regions and the narrow component corresponds to the amorphous region. The polymersalt complexes of lower salt concentration still show two overlapping signals but for the higher salt concentration $(x<100)$ the broad component is merged with the baseline (see figure 7). As the salt concentration increases the ${ }^{1} \mathrm{H}$ linewidth decreases sharply in a similar way to the decrease in the fraction of crystallinity as observed in DSC results and reaches a minimum at around $x=46$ and then increases (see figure 8). Further increase in ${ }^{1} \mathrm{H}$ linewidth with salt concentration though $X_{\mathrm{c}}$ decreases could be due to the decrease in polymer chain flexibility as indicated by an increase in $T_{\mathrm{g}}$ at higher salt concentration. The ${ }^{7} \mathrm{Li}$ spectra for $x=15,46$ and 100 are shown in figure 9. The ${ }^{7} \mathrm{Li}$ signals also change in an interesting fashion. The ${ }^{7} \mathrm{Li}$ signals become sharper as salt concentration increases and reaches a minimum at $x=46$ then broaden further (see figure 10). The decrease in ${ }^{7} \mathrm{Li}$ linewidth at lower salt concentration is consistent with the decrease in degree of crystallinity of the polymer with doping with salt and further increase in linewidth could be due to the increase in $\mathrm{Li}^{+}$ion-ion interaction resulting in decreased $\mathrm{Li}^{+}$motion.

\subsection{Ionic conductivity}

Pure PEG 2000 shows a low ionic conductivity $(\sim 1.67 \times$ $\left.10^{-9} \mathrm{~S} / \mathrm{cm}\right)$. This kind of conductivity was also observed for PEO (mol. wt. $4 \times 10^{6}$ ). This was explained due to either the presence of impurities, particularly $\mathrm{Ca}^{2+}$ and $\mathrm{Mg}^{2+}$ catalyst residues or an inherent proton generation process taking place in the polymer (Gray 1991). The ionic conductivity vs salt concentration (mole fraction) for (PEG) $\mathrm{LiClO}_{4}$ system at $300 \mathrm{~K}$ is shown in figure 11 . The ionic conductivity for the polymer-salt complexes increases with

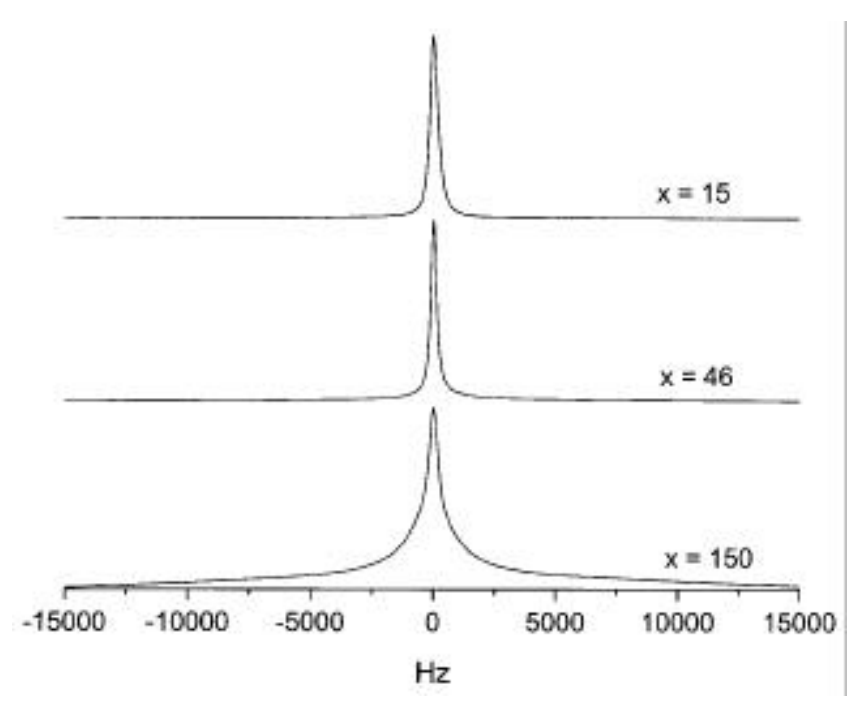

Figure 7. ${ }^{1} \mathrm{H}$ NMR spectra for $(\mathrm{PEG})_{x} \mathrm{LiClO}_{4}$. 
the salt concentration and reaches a peak $(\sigma=7 \cdot 27 \times$ $10^{-7} \mathrm{~S} / \mathrm{cm}$ ) at $x=46$ and then decreases. The initial increase in conductivity in the low concentration system is explained as due to the increase in charge carriers as the number of free cations increases and also a decrease in $X_{\mathrm{c}}$ with an increase in salt concentration. The further decrease in $\sigma$ though $X_{\mathrm{c}}$ decreases further at higher salt concentration could be due to an increase in the ion-ion interaction that impedes the motion of $\mathrm{Li}^{+}$and the stiffening of polymer chains as a result of cross links formed by cations and salt precipitation (Gray 1991).

Figure 12 shows the conductivity vs temperature plot of the $(\mathrm{PEG})_{x} \mathrm{LiClO}_{4}$ system. The ionic conductivity increases monotonically as the temperature rises and

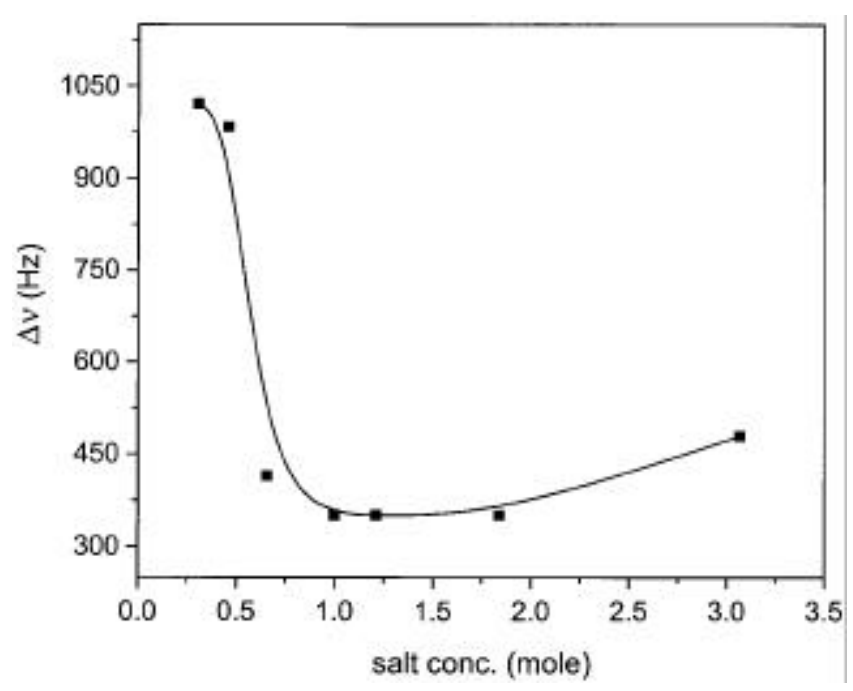

Figure 8. ${ }^{1} \mathrm{H}$ linewidth vs salt concentration plot for $(\mathrm{PEG})_{x}$ $\mathrm{LiClO}_{4}$.

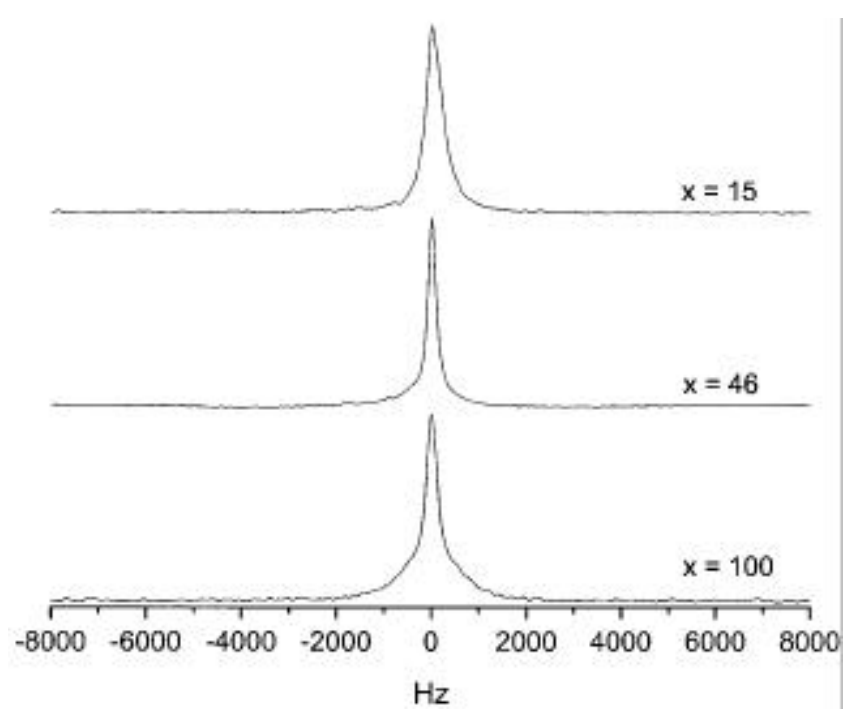

Figure 9. ${ }^{7} \mathrm{Li} \mathrm{NMR}$ spectra for $(\mathrm{PEG})_{x} \mathrm{LiClO}_{4}$. reaches a fairly high value $\left(\sim 10^{-4} \mathrm{~S} / \mathrm{cm}\right)$ for $x=46 \mathrm{com}-$ position just below the melting temperature. From the temperature dependence experiments, it is seen that there exists a temperature, $T_{\mathrm{c}}$, below which the conductivity plot has a curvature and above which it linearly and steeply rises till the melting of the samples. The region below $T_{\mathrm{c}}$ fits the empirical Vogel-Tammann-Fulcher (VTF) equation

$$
\sigma=\left(A / T^{1 / 2}\right) \exp \left[-B / k\left(T-T_{\mathrm{o}}\right)\right],
$$

(Vogel 1922; Fulcher 1925; Tamman and Hesse 1926), where $T$ is the absolute temperature and $T_{\mathrm{o}}$ the ideal glass transition temperature that is usually 30-50 degrees below $T_{\mathrm{g}}$, i.e. the temperature at which the configurational entropy vanishes, $B$ is an apparent activation energy which

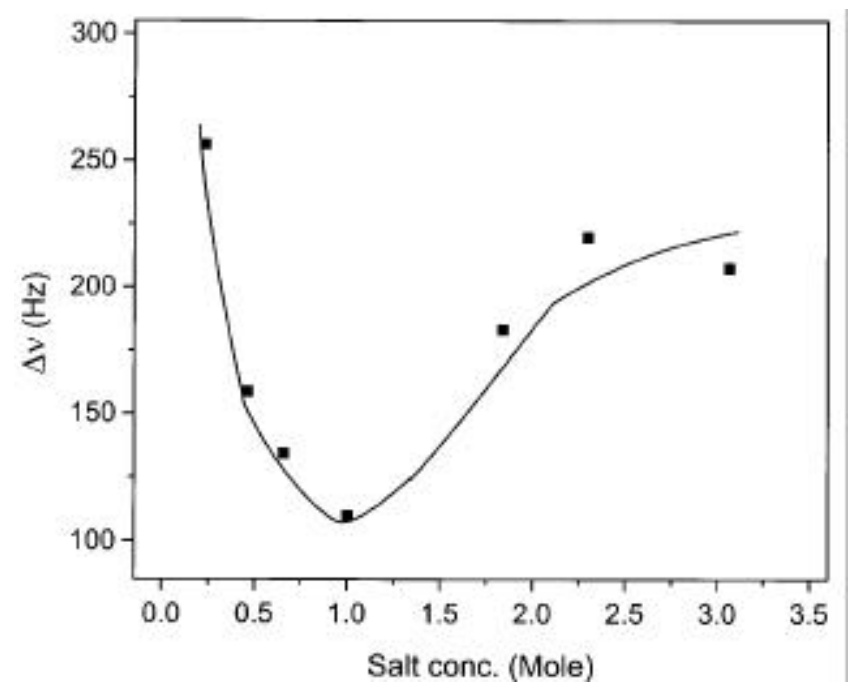

Figure 10. ${ }^{7} \mathrm{Li}$ linewidth vs salt concentration plot for $(\mathrm{PEG})_{x}$ $\mathrm{LiClO}_{4}$.

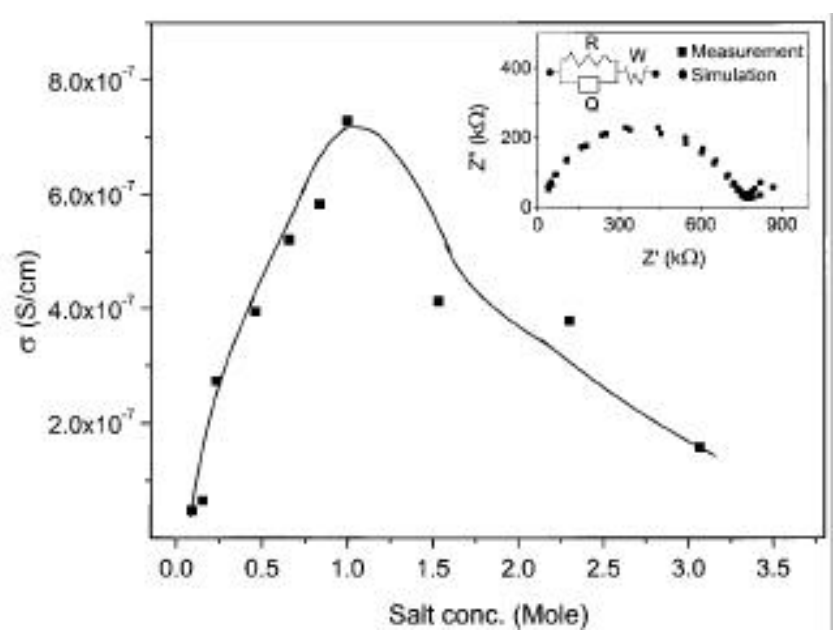

Figure 11. Conductivity vs salt concentration plot for $(\mathrm{PEG})_{x}$ $\mathrm{LiClO}_{4}$. The solid line is the guide to the eyes. Inset shows the typical impedance spectroscopy plot with equivalent circuit for $(\mathrm{PEG})_{46} \mathrm{LiClO}_{4}$. 
Table 2. Arrhenius and VTF equations fitting parameters for $(\mathrm{PEG})_{x} \mathrm{LiClO}_{4}$.

\begin{tabular}{lcccccc}
\hline$x$ value & $\begin{array}{c}\text { Salt } \\
(\text { mole })\end{array}$ & $E_{\mathrm{a}}(\mathrm{eV})$ & $T_{\mathrm{c}}(\mathrm{K})$ & $A\left(\mathrm{SK}^{0.5} \mathrm{~cm}^{-1}\right)$ & $B(\mathrm{eV})$ & $T_{\mathrm{o}}(\mathrm{K})$ \\
\hline 20 & $2 \cdot 300$ & $2 \cdot 57$ & 315 & 12.96 & $0 \cdot 13$ & $197 \cdot 9$ \\
46 & $1 \cdot 000$ & $2 \cdot 04$ & 310 & 1.732 & $0 \cdot 11$ & $195 \cdot 7$ \\
100 & 0.460 & $2 \cdot 12$ & 310 & 1.852 & $0 \cdot 12$ & $196 \cdot 4$ \\
300 & $0 \cdot 153$ & $2 \cdot 89$ & 315 & 1.692 & $0 \cdot 13$ & $199 \cdot 8$ \\
\hline
\end{tabular}

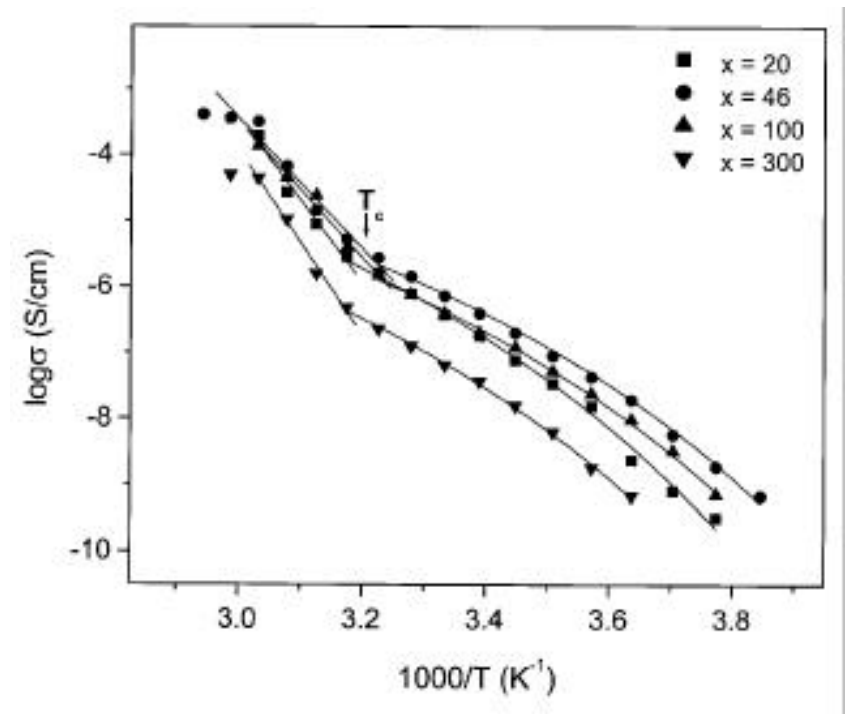

Figure 12. Conductivity vs temperature plot for $(\mathrm{PEG})_{x} \mathrm{LiClO}_{4}$.

is dependent on the free energy barrier opposing configurational rearrangements and $A$ a pre-exponential factor related to the number of carriers (Gibbs and DiMarzio 1958). The fitting parameter values are given in table 2. The Arrhenius equation

$$
\sigma=\sigma_{0} \exp \left[-E_{\mathrm{a}} / k T\right]
$$

where symbols have their usual meanings, fits the region above $T_{\mathrm{c}}$. The cross-over between Arrhenius and VTF behaviour of $\sigma(T)$ is widely reported and discussed in the literature (Ratner 1987; Binesh and Bhat 1998). This behaviour is rationalized by arguing that since VTF dependence is governed by the energy interval $\left(T-T_{\mathrm{o}}\right)$ and the Arrhenius dependence by the temperature $T$, for $T \gg T_{\mathrm{o}}$ the two should merge. A considerable amount of modelling effort has been devoted to understanding this behaviour in terms of the free volume theory of Cohen and Turnbull (1959). It is observed that $T_{\mathrm{o}}$ decreases as the salt concentration increases. Expectedly a correspondence between the ionic conductivity at $300 \mathrm{~K}$ and the activation energy, $E_{\mathrm{a}}$, is observed. $E_{\mathrm{a}}$ decreases and shows a minimum value at $x=46$ at the salt concentration that shows the maximum ionic conductivity and then increases with further increase in salt concentration (see table 2).

\section{Conclusions}

A new solid polymer electrolyte, $(\mathrm{PEG})_{x} \mathrm{LiClO}_{4}$, was prepared and characterized. XRD and IR confirm the formation of the polymer-salt complex. $(\mathrm{PEG})_{x} \mathrm{LiClO}_{4}$ shows interesting features as a function of salt concentration. The samples with higher concentration are softer and have rougher surfaces. The samples with lower salt concentration are harder, smoother and transparent. The glass transition temperature increases as the salt concentration increases due to the stiffening of the polymer chains by cross-linking them. $T_{\mathrm{m}}$ of SPEs is lower than the pure PEG 2000. The degree of crystallinity, $X_{c}$, was also observed to decrease with an increase in salt concentration. This reduction in $X_{\mathrm{c}}$ is attributed to the lower $T_{\mathrm{m}}$ and decrease in heat of melting, $\Delta Q_{\mathrm{m}}$, with increase in salt concentration. ${ }^{1} \mathrm{H}$ NMR results are consistent with the DSC results. ${ }^{7} \mathrm{Li}$ NMR results show the highest motion for $x=46$ composition. The salt concentration dependence of ionic conductivity shows a maximum $\left(7 \cdot 3 \times 10^{-7} \mathrm{~S} / \mathrm{cm}\right)$ at $x=46$. The ionic conductivity increases monotonically with temperature and reaches $\sigma \sim 10^{-4} \mathrm{~S} / \mathrm{cm}$ close to the melting temperature. The temperature dependence of the conductivity fits the Arrhenius and the VTF equations in different temperature ranges. The activation energy, $E_{\mathrm{a}}$, is the lowest for the maximum ionic conductivity salt concentration.

\section{Acknowledgements}

We thank the University Grants Commission, New Delhi, for financial support. One of the authors (TJKS) gratefully acknowledges the Council of Scientific and Industrial Research, New Delhi, for a research fellowship.

\section{References}

Armand M B, Chabagno J M and Duclot M 1978 Second international meeting on solid electrolytes (ed.) C A Vincent (Scotland: St. Andrews) p. 20

Berthier C, Gorecki W, Minier M, Armand M B, Chabagno J M and Rigaud P 1983 Solid State Ionics 1191

Binesh N and Bhat S V 1998 J. Polym. Sci. Part B: Polym. Phys. 361201

Boukamp B A 1989 EQUIVCRT program V 3.39 (The Netherlands: University of Twente)

Bruce P G and Vincent C A 1993 J. Chem. Soc. Faraday Trans. 893187 
Cohen M H and Turnbull D 1959 J. Chem. Phys. 311164

Fenton D E, Parker J M and Wright P V 1973 Polymer 14589

Fulcher G S 1925 J. Am. Ceram. Soc. 8339

Gibbs J H and DiMarzio E A 1958 J. Chem. Phys. 28373

Gray F M 1991 Solid polymer electrolyte-fundamentals and technological applications (New York: VCH Publishers, Inc.)

Hikichi K and Furuichi J 1965 J. Polym. Sci. A3 3003

Johansson A, Wendsjö A and Tegenfeldt J 1992 Electrochim. Acta 371487

Kelly I, Owen J R and Steele B C H 1984 J. Electroanal. Chem. 168467

Le Nest J F, Gandini A and Cheradane H 1988 Br. Polym. J. 20253

Li X and Hsu S L 1984 J. Polym. Sci., Polym. Phys. Ed. 221331

Ratner M A 1987 Polymer electrolyte reviews (eds) J R
MacCallum and C A Vincent (London: Elsevier Applied Science) Vol. 1, pp 185-195

Ratner M A and Shriver D F 1988 Chem. Rev. 88109

Shi J and Vincent C A 1993 Solid State Ionics 6011

Tamman G and Hesse W 1926 Z. Anorg. Allg. Chem. 19245

Tanzella F L, Baily W, Frydrych D, Farrington G C and Story H S 1981 Solid State Ionics 5681

Vincent C A 1987 Prog. Solid State Chem. 17145

Vogel H 1922 Phys. Z. 22645

Watanabe M, Togo M, Sanui K, Ogata N, Kobayashi T and Ohtaki Z 1984 Macromolecules 172908

Weston J E and Steele B C H 1982 Solid State Ionics 781

Whittingham M S, Weppner W and Schulz H (eds) 1988 Solid State Ionics 28-30 1

Wright P V 1975 Br. Polym. J. 7319 